\title{
Atomic Structure of $\mathrm{Cu}_{49} \mathrm{Hf}_{42} \mathrm{Al}_{9}$ Metallic Glass with High Glass-Forming Ability and Plasticity
}

\author{
Kai $\mathrm{Li}^{1,2,3} \cdot$ Yu-Jen Chou ${ }^{4}$ Fang-Liang Gao ${ }^{1,2,3} \cdot$ Guo-Qiang $\mathrm{Li}^{1,2,3}$
}

Received: 30 January 2018 / Revised: 21 March 2018 / Published online: 26 April 2019

(c) The Chinese Society for Metals (CSM) and Springer-Verlag GmbH Germany, part of Springer Nature 2019

\begin{abstract}
Electron diffraction was used to study the local atomic structure of $\mathrm{Cu}_{49} \mathrm{Hf}_{42} \mathrm{Al}_{9}$ metallic glasses (MGs). The amorphous nature of the MG was fully retained after the compression test. The partial radial distribution functions (PRDFs) of the MG structure obtained from the atomic model using reverse Monte Carlo and density functional theory optimization display that the peaks of the first nearest-neighbour distances for $\mathrm{Cu}-\mathrm{Cu}, \mathrm{Hf}-\mathrm{Cu}$ and $\mathrm{Hf}-\mathrm{Hf}$ atomic pairs were located at $2.56 \AA, 2.78 \AA$ and $3.23 \AA$, respectively. The wide distribution of PRDF for Hf-Hf atomic pair explained the high plasticity of the material.
\end{abstract}

Keywords Metallic glass $\cdot$ Radial distribution function $\cdot$ Electron diffraction $\cdot$ Density functional theory $\cdot$ Atomic structure

Recently, Cu-based metallic glasses (MGs) have been widely investigated due to their excellent glass-forming ability, good thermal stability, high mechanical properties at room temperature and relatively low-cost elements contained [1]. The bulk volume and lack of plasticity are the two major factors to limit the practical applications of $\mathrm{Cu}$-based MGs [2-4].

Several publications reported that $\mathrm{Cu}-\mathrm{Hf}-\mathrm{Al}$ alloys were suitable for using as structural materials in the engineering industry and motor car industry $[5,6]$ owing to their excellent mechanical properties, strength as well as corrosion and wear resistance [6-9]. $\mathrm{Cu}-\mathrm{Hf}-\mathrm{Al}$ metallic glass exhibited

Available online at http://link.springer.com/journal/40195

Fang-Liang Gao

gao.fl@mail.scut.edu.cn

$\triangle$ Guo-Qiang Li

msgli@scut.edu.cn

1 State Key Laboratory of Luminescent Materials and Devices, South China University of Technology, Guangzhou 510640, China

2 Engineering Research Center on Solid-State Lighting and Its Informationisation of Guangdong Province, South China University of Technology, Guangzhou 510640, China

3 Department of Electronic Materials, School of Materials Science and Engineering, South China University of Technology, Guangzhou 510640, China

4 Department of Materials, University of Oxford, Parks Road, Oxford OX1 3PH, UK high compressive fracture strength of 2260-2370 MPa and Young's modulus of 121-128 GPa [10]. At the same time, $\mathrm{Cu}-\mathrm{Hf}-\mathrm{Al}$ has good glass-forming ability and plasticity with the as-cast bulk metallic glass (BMG) $[11,12]$. It has been reported that a $\mathrm{Cu}_{49} \mathrm{Hf}_{42} \mathrm{Al}_{9}$ BMG rod with a casting diameter of $10 \mathrm{~mm}$ has been obtained and has shown considerable plasticity in compression tests up to 11-13\% [13]. It has been recognized that to understand the properties of MGs knowing its atomic structure is important. In the glass phase, where there is no long-range order, local atomic arrangements are believed to play an important role in defining its properties [14]. However, the above research mainly focuses on the exploration of improving the formation ability and ductility, by tailoring the combinations of the constituent elements or by controlling the cooling rate. The mechanism of its ductility cannot be revealed from the perspective of atomic structure. Detailed investigation of local atomic structure of $\mathrm{Cu}_{49} \mathrm{Hf}_{42} \mathrm{Al}_{9} \mathrm{MGs}$ is therefore critical to provide a basis for understanding its properties and building models for structure-properties correlation.

The radial distribution analysis is the standard way to study glassy structure using neutron or X-ray diffraction, which, however, requires averaging from a large volume of bulk material due to the low scattering cross section of neutron or X-ray. This shortcoming limits the application of neutron or X-ray diffraction in the study of metallic glass structures. Electron diffraction, on the contrary, provides a reliable tool to explore the local atomic structures of glassy materials. The structural information of metallic glass can be 
obtained even in small volumes as electron beam can be easily focused on chosen local small areas of glassy phases. In addition, the large electron scattering cross section ensures the strong enough scattering signal from the local small areas of glassy phases.

In this letter, we report the atomic structure of $\mathrm{Cu}_{49} \mathrm{Hf}_{42} \mathrm{Al}_{9}$ MG at the scale below $1 \mathrm{~nm}$ by employing the analysis of experimental reduced density function from electron diffraction based on the reverse Monte Carlo (RMC) refinements accompanied by density functional theory (DFT) calculations. The atomic structure of this material will help to advance our understanding of its properties and guide the development of plastic MGs.

The master $\mathrm{Cu}_{49} \mathrm{Hf}_{42} \mathrm{Al}_{9}$ MG ingots with the nominal composition (in at.\%) were prepared by arc melting under a Ti-gettered argon atmosphere in a water-cooled copper crucible. The master alloy was remelted several times to ensure compositional homogeneity and finally cast in the copper mould to form $\mathrm{Cu}_{49} \mathrm{Hf}_{42} \mathrm{Al}_{9} \mathrm{MG}$ ingots of $3 \mathrm{~mm}$ in diameter. The as-cast $\mathrm{Cu}_{49} \mathrm{Hf}_{42} \mathrm{Al}_{9} \mathrm{MG}$ ingots were then cut to 2-mm-high rods with the both loading surfaces polished to be parallel to accuracy better than $10 \mu \mathrm{m}$. The MG rods were applied with compression test at room temperature with a loading strain rate of $1 \times 10^{-4} \mathrm{~s}^{-1}$ to check the plasticity. Both the as-cast and compressed $\mathrm{Cu}_{49} \mathrm{Hf}_{42} \mathrm{Al}_{9}$ samples were studied by transmission electron microscopy (TEM).

For TEM sample preparation, $\mathrm{Cu}_{49} \mathrm{Hf}_{42} \mathrm{Al}_{9}$ MGs were sliced into 3-mm discs, ground to a thickness of $\sim 40 \mu \mathrm{m}$ and ion-milled in a precision ion polishing system operating at $3.0 \mathrm{kV}$ (PIPS, Gatan, Pleasanton, CA) till the appearance of a tiny hole in the sample. For final thinning, the samples were moved to a low-angle ion milling system with a liquid nitrogen cooling stage working at a low voltage of $300 \mathrm{~V}$ (Fischione 1010 Low Angle Ion Milling \& Polishing System), preventing any possible structural alteration caused by the high-energy ion beam of PIPS.

The electron diffraction was carried out on a JEOL 3000F field emission gun TEM operating at $300 \mathrm{kV}$. Selected area electron diffraction (SAED) was used to collect the electron diffraction patterns from different thin areas in the sample. The selected area was, in each case, a disc of diameter about $100 \mathrm{~nm}$ on the sample. To avoid multiple scattering, only very thin areas with $t / \lambda \leq 0.4$ were used for SAED, where $t$ is the thickness of the selected area and $\lambda$ is the mean free path for inelastic electron scattering for $\mathrm{Cu}_{49} \mathrm{Hf}_{42} \mathrm{Al}_{9}$ MG. $t / \lambda$ was obtained in situ by electron energy loss spectrum (EELS) at the same locations where SAED patterns were collected, via the formula $t / \lambda=\ln \left(I_{\mathrm{T}} / I_{\mathrm{ZL}}\right)$, where $I_{\mathrm{T}}$ is the total intensity in the EELS spectrum and $I_{\mathrm{ZL}}$ is the intensity in the elastic zero-loss peak. The diffraction patterns were recorded on a $1000 \times 1000$ pixel CCD camera, corrected for dark current and pixel sensitivity. All the diffraction patterns were accurately calibrated by setting up a protocol of constant lens settings for the TEM, which enabled the calibration against an Au polycrystalline sample to be maintained to better than $1 \%$ accuracy. Using a camera length of $15 \mathrm{~cm}$, diffraction patterns were collected up to a scattering vector of $q(=4 \pi \sin \theta / \lambda) \approx 220 \mathrm{~nm}^{-1}$.

Uniaxial compressive stress-strain tests were carried out on five $\mathrm{Cu}_{49} \mathrm{Hf}_{42} \mathrm{Al}_{9} \mathrm{MG}$ samples. The compressive strain was $10-13.0 \%$, which was similar to the study of Jia et al. [13]. The corresponding uniaxial compressive stress-strain curve is shown in Fig. 1. As displayed in the inserted optical micrograph in Fig. 1, many shear bands were seen in the compressed $\mathrm{Cu}_{49} \mathrm{Hf}_{42} \mathrm{Al}_{9}$ under the optical microscopy which reflects the large plasticity of this MG [15]. Here, we name the areas other than shear bands in the MG as "matrix".

Careful observation of high-resolution transmission electron microscopy (HRTEM) is carried out on the compressed sample. No detectable crystalline clusters were observed in either shear bands or the matrix of $\mathrm{Cu}_{49} \mathrm{Hf}_{42} \mathrm{Al}_{9} \mathrm{MG}$, indicating that the high plasticity of this $\mathrm{MG}$ is originated from the amorphous structure itself, other than introducing some composite structures or nano-crystallites as adopted by some MGs to improve the plasticity [16, 17]. As a demonstration, Fig. 2 shows a typical HRTEM micrograph from the matrix, with the SAED pattern collected from this local area.

The electron diffraction data from the matrix of the compressed sample were converted to radial distribution functions (RDFs) by techniques developed by Cockayne et al. [18, 19]. The reduced intensity function $\varphi(q)$ was obtained by subtracting the theoretical atomic scattering from the total diffraction intensity $I(q)$ using the following formula:

$\phi(q)=\left[\frac{I(q)-N<f(q)^{2}>}{N\langle f(q)\rangle^{2}}\right] q$,

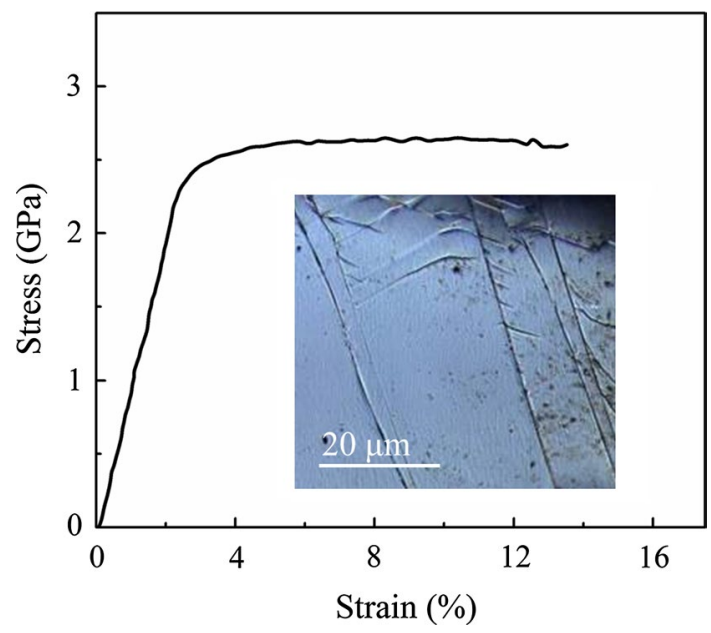

Fig. 1 Uniaxial compressive stress-strain curve of $\mathrm{Cu}_{49} \mathrm{Hf}_{42} \mathrm{Al}_{9}$ MG. The inset is an optical micrograph of $\mathrm{Cu}_{49} \mathrm{Hf}_{42} \mathrm{Al}_{9} \mathrm{MG}$ after the compression test, showing multiple shear bands 


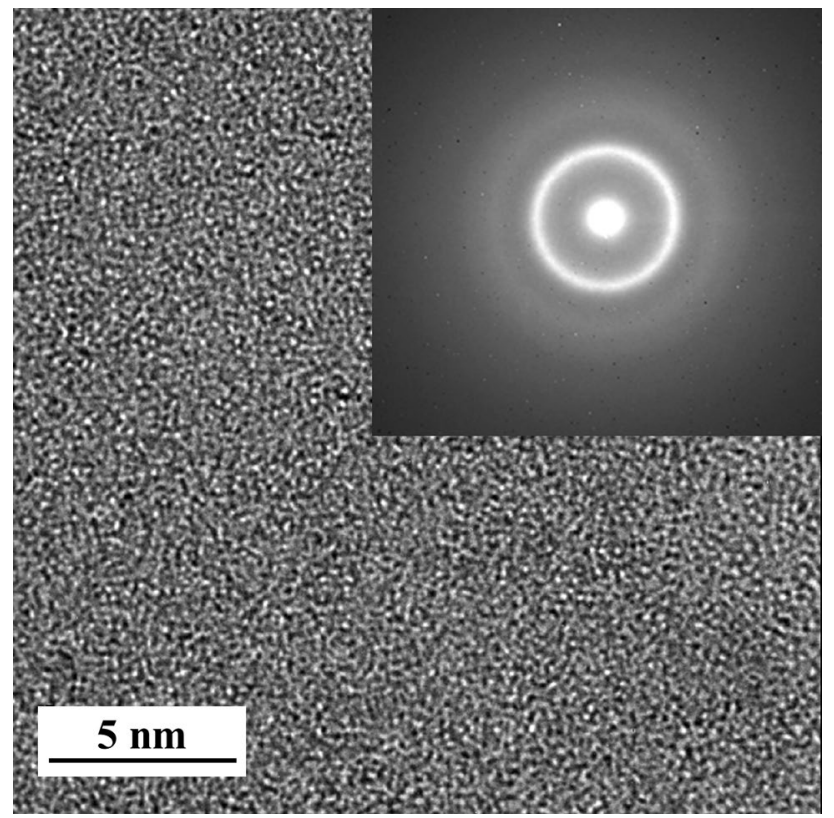

Fig. 2 A typical HRTEM image from the matrix of plastically deformed $\mathrm{Cu}_{49} \mathrm{Hf}_{42} \mathrm{Al}_{9}$ MG. No crystalline structure is detected. The inset is the SAED pattern collected from this local area

where $<f^{2}>=\sum_{i}\left(N_{i} f_{i}^{2} / N\right)$ and $<f>^{2}=\left(\sum\left(N_{i} f_{i}\right)^{2}\right) / N^{2}$ with $N=\sum_{i} N_{i}$ using the electron scattering factors $f_{i}(q)$ of Kirkland, where the element $i$ has $N_{i}$ atoms contributing. $N$ is treated as the only fitting parameter by setting $I(q)=N<f(q)^{2}>$ at large $q$. We should be noticing that intensity profiles up to scattering angles as high as $q=220 \mathrm{~nm}^{-1}$ are recorded well above the background intensity level, which is much higher than the typical $120-150 \mathrm{~nm}^{-1}$ level in X-ray and neutron diffraction [20-22]. This means that we can obtain a higher spatial resolution in the real lattice space as compared to the previous diffraction experiments. It is seen from Fig. 3a that the fit of $I(q)$ to $N<f(q)^{2}>$ at even high $q$ is excellent and that $I(q)$ oscillates around
$N<f(q)^{2}>$ at low $q$, giving confidence that the single scattering approximation is satisfied. The corresponding reduced intensity function $\varphi(q)$ is shown in Fig. 3b, and the Fourier transform of $\varphi(q)$ leads to reduced density function $G(r)$ as shown in Fig. 3c. Electron diffraction data were also collected from as-cast $\mathrm{Cu}_{49} \mathrm{Hf}_{42} \mathrm{Al}_{9} \mathrm{MG}$ and converted to $\mathrm{RDF}$, which is found to be identical to the one from the matrix of the compressed sample (Fig. 3c). It is indicative that the matrix structure is the same as the as-cast glassy structure. In other words, the plastic deformation of the MG is only confined in shear bands.

A cubic cell of the structure contains 100 atoms with a lattice parameter of $12.5 \AA$, corresponding to a density of $9.0 \mathrm{~g} \mathrm{~cm}^{-3}$, slightly lower than the actual density of $9.66 \mathrm{~g} \mathrm{~cm}^{-3}$. And then, periodic boundary conditions in three dimensions were first constructed. RMC simulation was used to refine the atomic structure by comparing the experimental reduced scattering intensity with that computed for the atomic model of the material. The structure resulting from the RMC procedure was optimized using DFT software. The optimizations were performed using the ab initio VASP codes [23, 24]. Perdew-Wang exchange-correlation functional containing general gradient approximation (GGAPW91) was used to describe the electron exchange correlation and the convergence criteria of geometry optimization. The relaxation of the structures continued until the energy changes of ions and electrons were less than $1 \times 10^{-4}$ and $1 \times 10^{-5} \mathrm{eV}$. The structure refined by RMC and DFT leaded to an energetically stable atomic structure. Residual pressures in the relaxed structures were found to be small, about $-50 \mathrm{kbar}$, suggesting that the selected density is close to the optimal value for the alloy and justifying the fixed volume relaxation. Figure 4a shows the curve of RDF after DFT is very close to that from the experimental data.

Figure $4 \mathrm{~b}$ shows the partial radial distribution functions (PRDFs) obtained from this DFT-optimized structure. We note that the first nearest-neighbour distance for $\mathrm{Hf}-\mathrm{Cu}$ atomic pairs is located at $2.78 \AA$ that accounts for
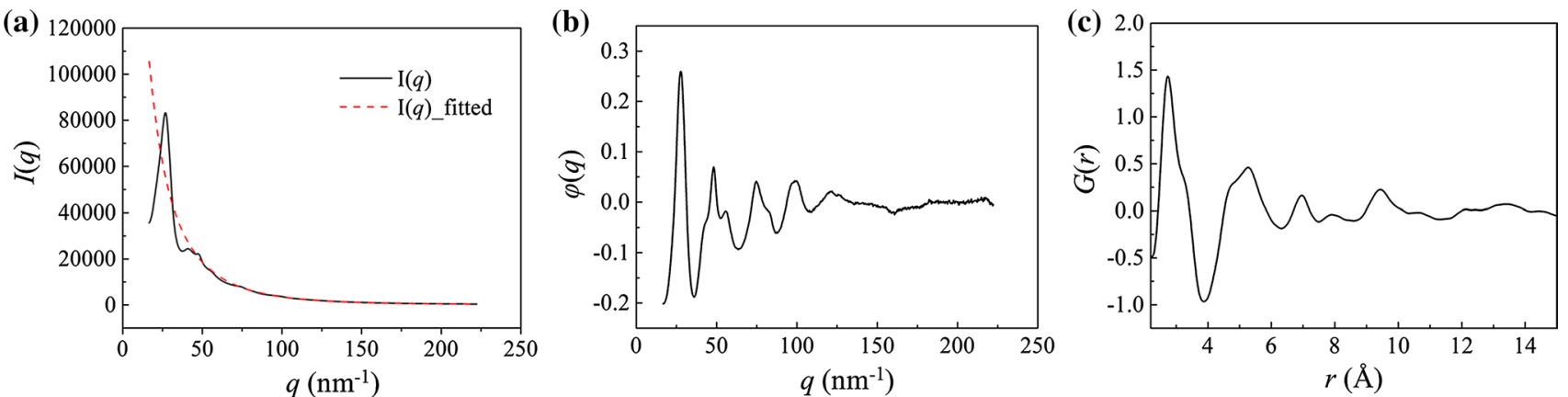

Fig. 3 a $I(q)$ from the matrix of plastically deformed $\mathrm{Cu}_{49} \mathrm{Hf}_{42} \mathrm{Al}_{9} \mathrm{MG}, I(q)$ fitted from independently scattering atoms of the same material, $\mathbf{b}$ reduced intensity $\varphi(q), \mathbf{c} \mathrm{RDF}$ at the examined position in $\mathrm{Cu}_{49} \mathrm{Hf}_{42} \mathrm{Al}_{9} \mathrm{MG}$ 

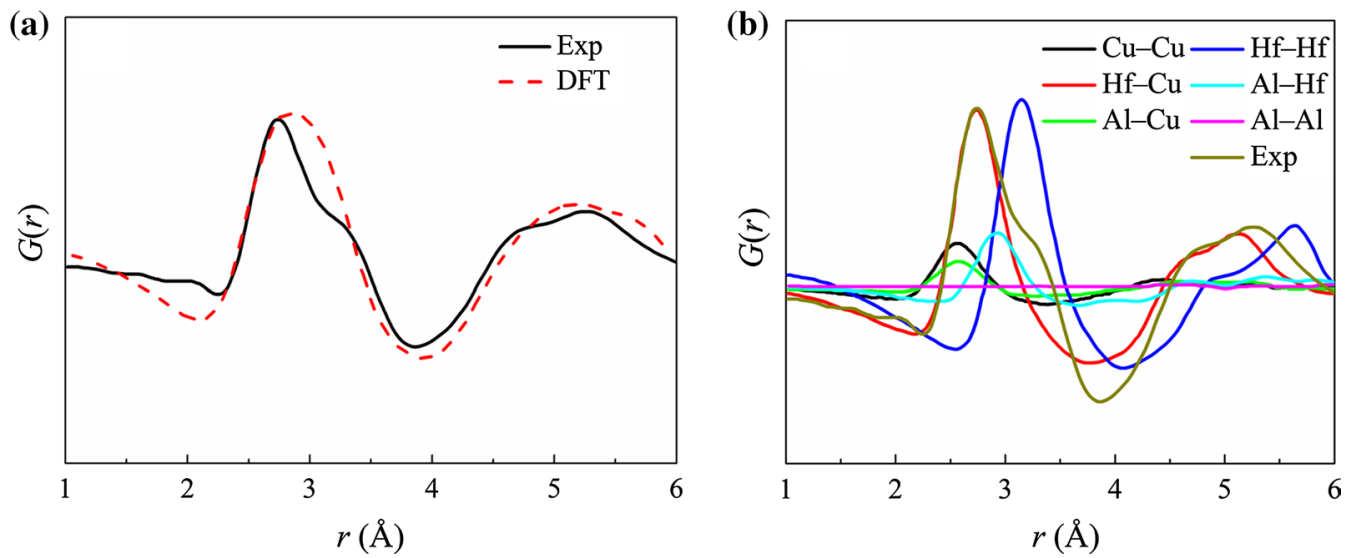

Fig. 4 a RDF curves obtained from simulation after DFT and experiment, b PRDFs obtained from the DFT-optimized structure

the origin of the main peak of the $G(r)$ between 2 and $4 \AA$, while the first nearest-neighbour distance for $\mathrm{Hf}-\mathrm{Hf}$ atomic pairs is situated at about $3.23 \AA$ which forms the shoulder of this range in $G(r)$. The second nearest neighbours from $\mathrm{Hf}-\mathrm{Cu}$ and $\mathrm{Hf}-\mathrm{Hf}$ atomic pairs also contribute the most to the second main peak of the $G(r)$ between 4 and $6 \AA$. The $\mathrm{Cu}-\mathrm{Cu}$ atomic pairs with the nearest-neighbour distance at $2.56 \AA$, on the contrary, do not show strong PRDF peaks given the high concentration of $\mathrm{Cu}$ in this ternary MG. This suggests that the chance to form $\mathrm{Cu}-\mathrm{Cu}$ pairs, or in other words, the chance of one $\mathrm{Cu}$ atom neighbouring another, is very little in this MG. $\mathrm{Cu}$ atoms are mainly neighbouring to $\mathrm{Hf}$ atoms, which can be deduced from the strong PRDF peaks of $\mathrm{Hf}-\mathrm{Cu}$ atomic pairs. Actually, this is understandable considering the radii of the two elements, $1.28 \AA$ for $\mathrm{Cu}$ and $1.59 \AA$ for Hf. Hf atom is about $24.21 \%$ larger in radius than $\mathrm{Cu}$ atom, so that the small $\mathrm{Cu}$ atoms are more likely to be fit into the interstitions among the much bigger Hf atoms during the formation of glassy structure. This complex structure is not conducive to the rearrangement of atoms in the cooling process and is conducive to form the amorphous structure of metallic glass. Figure $4 \mathrm{~b}$ shows the wide $\mathrm{Hf}-\mathrm{Hf}$ band distribution of PRDF, which means that the bond lengths of Hf-Hf are a set of array, which all can form stable atomic links.

The HRTEM and RDF of the sample matrix and those of the shear band are shown in Fig. 5. The RDFs of the matrix and shear band are represented in magenta and blue, respectively. The contrast of shear band and undeformed matrix is obviously different. The contrast of shear band is obviously higher than that of matrix. There are no obvious lattice striations in the shear band or in the undeformed matrix outside the shear band. From the figure, the peak of the nearest-neighbour distance in the shear band RDF is wider and shorter than that in the matrix RDF, indicating the reduced probability of the atom appearing in the shear band at this distance, i.e. more free volume exists in the shear band. The RDF of the matrix presents peak splitting and thus a shoulder feature, while the shoulder feature is less evident in the RDF of the shear band. Concurrently, the RDF

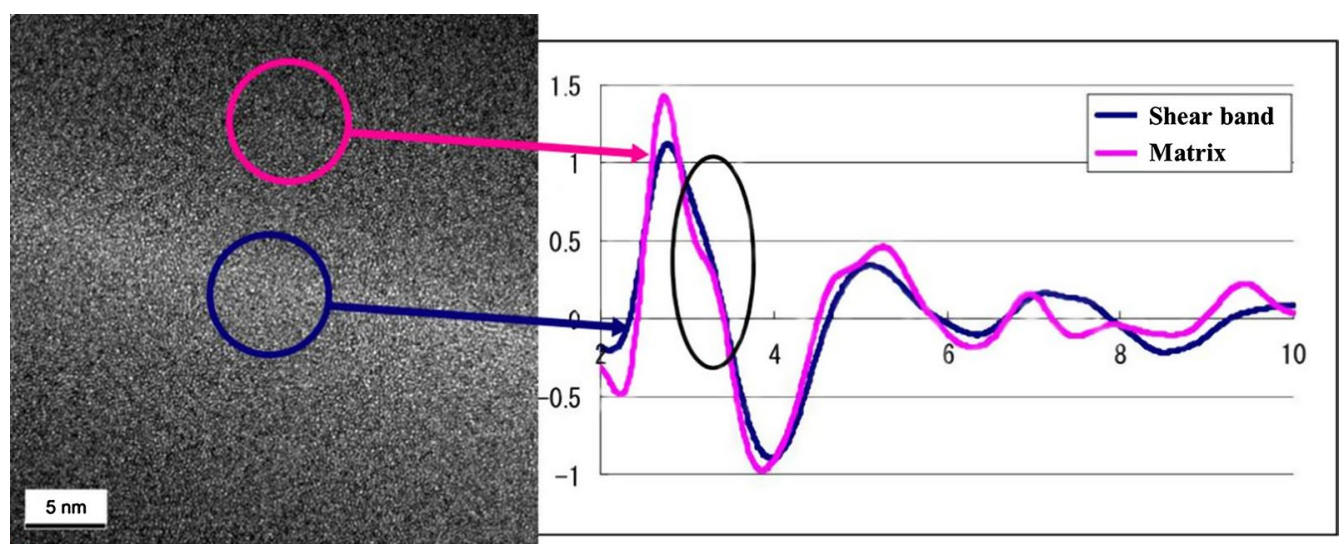

Fig. 5 Comparison of the HRTEM and RDF of the matrix and those of the shear band 
peak of the shear band region is right-shifted in the $3-4-\AA$ interval. Therefore, in the shear band of the $\mathrm{Cu}_{49} \mathrm{Hf}_{42} \mathrm{Al}_{9}$ $\mathrm{MG}$, the microstructure is affected by the stress, and the nearest-neighbour distances of some atoms in the shear band are elongated compared with those of atoms in the matrix.

From Fig. 1, some dense shear bands are present in the compressed $\mathrm{Cu}_{49} \mathrm{Hf}_{42} \mathrm{Al}_{9}$ sample, indicating that the $\mathrm{Cu}_{49} \mathrm{Hf}_{42} \mathrm{Al}_{9}$ MG undergoes a certain degree of plastic deformation in some localized regions. Under the action of external stress, the interaction of many atomic transitions produces a macroscopic plastic deformation in a MG and the rearrangement of atom positions [25]. From the atomiclevel structure perspective, Lee et al. [26, 27] revealed that the plasticity in an amorphous alloy strongly depends on its atomic packing density (i.e. the free volume fraction). Under external stress, the structural differences of the microregions result in the shear relaxation of different volume units to not be completely consistent. In the MG, some microregions contain more free volume, and under external stress, shear strain occurs in these microregions. At room temperature, these local strains cannot dissipate efficiently; they eventually form continuous deformation zones in the microregions of the material so that dense shear bands appear in some localized regions of the MG matrix [28]. Hf-Hf atomic pairs enable the MG to still form a stable structure. Therefore, the shear band formation rapidly proliferates at lower external stresses far below the fracture stress, and the ductility is improved [29].

The local atomic structure of $\mathrm{Cu}_{49} \mathrm{Hf}_{42} \mathrm{Al}_{9}$ MG which exhibits the plasticity up to $10-13 \%$ was studied by TEM. HRTEM images revealed that the MG kept completely amorphous even after the plasticity deformation test. RDF analysis indicates that the matrix of the compressed sample shares the same structure as the as-cast MG, suggesting that the plastic deformation of the MG is confined in shear bands. Reduced intensity function $\varphi(q)$ from $\mathrm{Cu}_{49} \mathrm{Hf}_{42} \mathrm{Al}_{9}$ shows more and stronger oscillations than other reported MGs, indicating a much ordered structure than the normal MGs. PRDFs from the atomic structure optimized by RMC and DFT against the electron diffraction data exhibit the first nearest-neighbour distances for $\mathrm{Cu}-\mathrm{Cu}, \mathrm{Hf}-\mathrm{Cu}$ and $\mathrm{Hf}-\mathrm{Hf}$ atomic pairs located at $2.56 \AA, 2.78 \AA$, and $3.23 \AA$, respectively. $\mathrm{Cu}-\mathrm{Cu}$ pairs have a much less contribution to RDF compared with $\mathrm{Hf}-\mathrm{Cu}$ and $\mathrm{Hf}-\mathrm{Hf}$, indicating that $\mathrm{Cu}$ atoms are mainly filled into the interstitions between $\mathrm{Hf}$ atoms in the glassy structure. HRTEM shows that the contrast of the MG shear band is significantly higher than that of the matrix, and both the sample matrix and the shear band are completely amorphous. The first adjacent distance of RDF in the shear band is wider than that in the matrix, indicating that the first adjacent distance of some atoms in the shear band is longer than that in the matrix. The
PRDF of Hf-Hf atomic pair has a wide distribution. These microscopic structures might account for the unusually high plasticity of $\mathrm{Cu}_{49} \mathrm{Hf}_{42} \mathrm{Al}_{9}$ MG.

Acknowledgements This work was supported by the National Science Fund for Excellent Young Scholars of China (No. 51422203), the National Natural Science Foundation of China (Nos. 51572091 and 51372001), the Natural Science Foundation of Guangdong Province, China (No. 2018A030313395), the China Postdoctoral Science Foundation (No. 2017M610522), the Science and Technology Program of Guangzhou, China (No. 201604046027), the Fundamental Research Funds for the Central Universities (No. 2017BQ035) and the fund of the State Key Laboratory of Solidification Processing in NWPU (No. SKLSP201716).

\section{References}

[1] K. Yang, X.H. Fan, B. Li, Y.H. Li, X. Wang, X.X. Xu, Acta Metall. Sin. (Engl. Lett.) 31, 290 (2018)

[2] Y. Dong, R. Wunderlich, J. Biskupek, Q.P. Cao, X.D. Wang, D.X. Zhang, J.Z. Jiang, H.J. Fecht, Scripta Mater. 137, 94 (2017)

[3] J.J. Liu, R. Li, L.X. Fang, J. Wang, T. Zhang, Acta Metall. Sin. (Engl. Lett.) 29, 129 (2016)

[4] F.F. Wu, S.S. Jiang, R.D. Zhao, Q. Zhou, G.A. Zhang, X.F. Wu, Mater. Sci. Eng., A 646, 272 (2015)

[5] B. Zhang, Y.G. Chen, H.B. Guo, J. Alloys Compd. 582, 496 (2014)

[6] E. Nagy, V. Rontó, J. Sólyom, A. Roósz, J. Phys: Conf. Ser. 144, 012035 (2009)

[7] J.Y. Mo, H.S. Liu, Y. Zhang, M.Z. Wang, L. Zhang, B.Z. Liu, W.M. Yang, J. Non-Cryst, Solids 464, 1 (2017)

[8] J.C. Zhang, C. Chen, Q.X. Pei, Q. Wan, W.X. Zhang, Z.D. Sha, Mater. Des. 77, 1 (2015)

[9] X. Huang, Z. Ling, Y.J. Wang, L.H. Dai, Intermetallics 75, 36 (2016)

[10] A. Inoue, W. Zhang, J. Saida, Mater. Trans. 45, 1153 (2004)

[11] C.Y. Haein, J. Korean Phys. Soc. 60, 485 (2012)

[12] E.S. Park, J.S. Kyeong, D.H. Kim, Scripta Mater. 57, 49 (2007)

[13] P. Jia, H. Guo, Y. Li, J. Xu, E. Ma, Scripta Mater. 54, 2165 (2006)

[14] G. Wang, S. Pauly, S. Gorantla, N. Mgttern, J. Eckert, J. Mater. Sci. Technol. 30, 609 (2014)

[15] W.L. Ma, Y.L. Xu, B. Shi, J.G. Li, J. Mater. Sci. Technol. 33, 99 (2017)

[16] T.M. Chung, S.R. Jian, P.J. Hsieh, Metals 6, 216 (2016)

[17] Y.P. Jiang, K. Qiu, Mater. Des. 65, 410 (2015)

[18] D.J.H. Cockayne, D.R. McKenzie, Acta Cryst. A 44, 870 (1988)

[19] G.Q. Li, K.B. Borisenko, Y. Chen, D. Nguyen-Manh, E. Ma, D.J.H. Cockayne, Acta Mater. 57, 804 (2009)

[20] X.H. Wang, A. Inoue, J.F. Zhao, F.L. Kong, S.L. Zhu, I. Kaban, M. Stoica, S. Oswald, C. Fan, E. Shalaan, F. Al-Marzouki, J. Eckert, F.X. Yin, Q. Li, J. Alloy. Compd. 739, 1104 (2018)

[21] L. Tang, T.Q. Wen, N. Wang, Y. Sun, F. Zhang, Z.J. Yang, K.M. Ho, C.Z. Wang, Phys. Rev. Mater. 2, 03360 (2018)

[22] D. Ma, A.D. Stoica, X.L. Wang, Nat. Mater. 8, 30 (2009)

[23] G. Kresse, J. Hafner, Phys. Rev. B 48, 13115 (1993)

[24] G. Kresse, J. Hafner, Phys. Rev. B 49, 14251 (1994)

[25] F. Spaepen, Acta Metall. 25, 407 (1977)

[26] J.C. Lee, K.W. Park, K.H. Kim, E. Fleury, B.J. Lee, M. Wakeda, Y. Shibutani, J. Mater. Res. 22, 3087 (2007)

[27] Y.Q. Cheng, H.W. Sheng, E. Ma, Phys. Rev. B 78, 014207 (2008)

[28] P.S. Steif, F. Spaepen, J.W. Hutchinson, Acta Metall. 30, 447 (1982)

[29] T. Wada, A. Inoue, A.L. Greer, Appl. Phys. Lett. 86, 251907 (2005) 